\title{
Modeling the Impact of Carbon Dioxide on Marine Plankton
}

\author{
Sajib Mandal ${ }^{1 *}$, M. S. Islam ${ }^{1}$, M. H. A. Biswas ${ }^{2}$ \\ ${ }^{1}$ Department of Mathematics, Bangabandhu Sheikh Mujibur Rahman Science and Technology \\ University, Gopalganj-8100, \\ Bangladesh. \\ ${ }^{2}$ Mathematics Discipline, Khulna University, \\ Bangladesh.
}

\begin{abstract}
Marine plankton is the main absorber of environmental carbon dioxide. According to scientists, the marine plankton not only absorbs approximately $30-50 \%$ carbon dioxide but also supplies about $70 \%$ oxygen of the atmosphere. Carbon dioxide helps the marine plankton to carry on the photosynthetic activity which promotes the growth of the planktonic population. As a result, the increasing plankton absorbs more saturated carbon dioxide and supplies more oxygen. In this article, a mathematical model has been proposed to describe the contribution of carbon dioxide on marine plankton. We have analyzed the model both analytically and numerically. The study discloses natural behaviors along with mathematical modeling.
\end{abstract}

Keywords-Mathematical modeling, Analysis, Simulation, Global warming.

\section{INTRODUCTION}

$\mathrm{M}$ ARINE ecosystem is a great source of oxygen and a great system of absorbing carbon dioxide $\left(\mathrm{CO}_{2}\right)$. About $70 \%$ of the oxygen of the atmosphere [1] is produced by phytoplankton in oceans due to photosynthetic activity on which total lives on the earth depends mostly. On the other hand, Scientists believe that the oceans currently absorb 30$50 \%$ of the $\mathrm{CO}_{2}$ produced in the atmosphere [1]. The capacity for absorbing $\mathrm{CO}_{2}$ and production of $\mathrm{O}_{2}$ in the oceans depends on the number of phytoplankton.

Phytoplankton mainly depends on photosynthetic activity and nutrients and photosynthetic activity depends on $\mathrm{CO}_{2}$, light, temperature, the density of the water layer surface. When $\mathrm{CO}_{2}$, light, and temperature rise, photosynthetic activity proportionally increases but reverses with the density of water layer surface and dissolved $\mathrm{O}_{2}$. Zooplankton depends on phytoplankton and dissolved $\mathrm{O}_{2}$ in water and the number of zooplankton raises proportionally with the number of phytoplankton and dissolved $\mathrm{O}_{2}$ and inversely proportional to the density of dissolved $\mathrm{CO}_{2}$. On the other hand, the density of
$\mathrm{CO}_{2}$ and $\mathrm{O}_{2}$ will change due to the change of planktons because they are also dependent on planktons. Thus they make an interacting dependency cycle among themselves.

Mandal et al. [2] developed a mathematical model to describe the effect of atmospheric temperature on the growth of phytoplankton. Destania et al. [3] discussed the growth of plankton influenced by oxygen deficit and in which the effect of nutrients on the plankton was also discussed. Khare [4] discussed the effect of the depleting dissolved oxygen on the existence of interacting planktonic population in which nutrient was also considered. Mandal et al. [5] illustrated the potential impact of greenhouse gases on coastal living beings through mathematical modeling. Sekerci and Petrovskii [1] discussed the effect of oxygen on the plankton. Some other ecological modeling [6]-[8] studies involving phytoplankton, zooplankton, and nutrients, and some other modeling [9]-[11] studies the effect of dissolved oxygen on plankton ecosystem.

In this work, the effect of carbon dioxide on the plankton (phytoplankton and zooplankton) has been discussed. The effect to the plankton due to change of temperature, light, and nutrient is very slow with respect to time. In this study, the interaction of the density of carbon dioxide, the density of phytoplankton, the density of zooplankton, and the concentration of dissolved oxygen are considered, and other interactions are neglected for the simplicity of calculations.

\section{MOdEL FORMULATION}

To formulate the mathematical modeling of the effect of carbon dioxide to the plankton-ecosystem, a system of nonlinear differential equations involving four variables is considered and the variables are the density of Carbon dioxide $(C)$, the density of Phytoplankton $(P)$, the density of Zooplankton $(Z)$, the concentration of dissolved Oxygen $(D)$. 


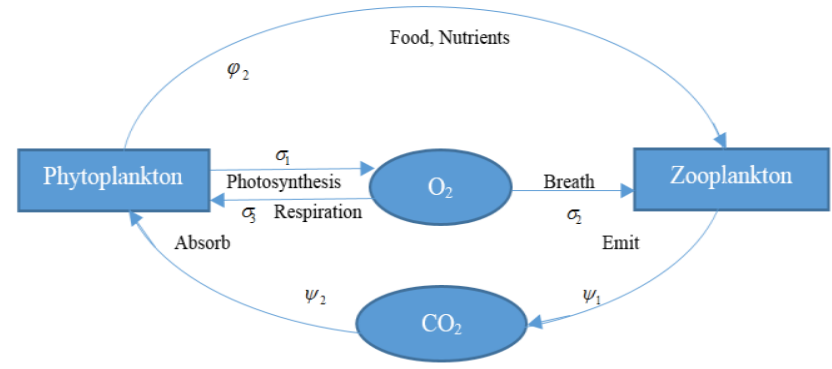

Fig.1 Flowchart of the system

Fig. 1 shows that phytoplankton serves the food and oxygen to zooplankton, and zooplankton serves the carbon dioxide to phytoplankton for photosynthetic activity. Thus they make an ecosystem among them and the interaction can be represented by a system of mathematical modeling bellowed:

From the above discussion, the ecosystem can be represented by the system of non-linear ordinary differential equations as follows:

$$
\begin{aligned}
& \frac{d C}{d t}=m+\psi_{1} C Z-\psi_{2} C P-\psi_{3} C \\
& \frac{d P}{d t}=\frac{\beta_{1} C P}{\alpha_{1}+D_{0}-D}-\varphi_{1} P-\varphi_{2} P Z \\
& \frac{d Z}{d t}=\frac{\beta_{2} P Z}{\alpha_{2}+D_{0}-D}-\gamma_{1} Z \\
& \frac{d D}{d t}=n+\sigma_{1} P C-\sigma_{2} D Z-\sigma_{3} D P-\sigma_{4} D
\end{aligned}
$$

with the initial conditions

$$
C(0) \geq 0, P(0) \geq 0, Z(0) \geq 0, D(0) \geq 0
$$

Brief description of the parameters used in the model are:

$m$ : $\quad$ Saturated rate of $\mathrm{CO}_{2}$ at getting into the water

$\psi_{1}$ : The production rate of $\mathrm{CO}_{2}$ by Zooplankton.

$\psi_{2}$ : The absorbing rate of $\mathrm{CO}_{2}$ by phytoplankton

$\psi_{3}$ : Natural depleting rate of $\mathrm{CO}_{2}$

$\varphi_{1}: \quad$ Natural death rate of phytoplankton

$\varphi_{2}$ : The predation rate of zooplankton

$\alpha_{1}: \quad$ Saturation constants

$\beta_{1}$ : $\quad$ Positive proportional constants

$D_{0}$ : The saturation value of dissolved oxygen

$\gamma_{1}: \quad$ Natural death rate of zooplankton

$\alpha_{2}$ : Saturation constants

$\beta_{2}$ : Positive proportional constants

$n$ : Concentration of dissolved oxygen enters into the system from a variety of sources

$\sigma_{1}$ : $\quad$ Producing rate of $\mathrm{O}_{2}$ by phytoplankton

$\sigma_{2}$ : Decreasing rate of $\mathrm{O}_{2}$ due to the breathing of zooplankton

$\sigma_{3}$ : Decreasing rate of $\mathrm{O}_{2}$ due to respiration of phytoplankton

$\sigma_{4}: \quad$ Natural depleting rate of $\mathrm{O}_{2}$

\section{ANALYTICAL ANALYSIS}

In the analytical section, we perform the positivity test of the dynamical variables, stability analysis at equilibrium points, and numerical simulation [12]-[14].

\section{A. Positivity Test}

Lemma. Let $\quad C(0) \geq 0, P(0) \geq 0, Z(0) \geq 0, D(0) \geq 0 \quad$ and $C(0), P(0), Z(0), D(0) \in R_{4}^{+}$then the solutions of the system of the model are positive.

Proof. To verify the lemma for the model, we have used the system (1)-(4). To find the positivity of (1), we have

$$
\begin{aligned}
& \frac{d C}{d t} \geq m+\psi_{1} C Z-\psi_{2} C P-\psi_{3} C \\
& \frac{d C}{d t}+b C \geq m, \text { where } b=-\psi_{1} Z+\psi_{2} P+\psi_{3}
\end{aligned}
$$

So the integrating factor is $e^{\int b d t}=e^{b t}$

Multiplying the integrating factor on both sides of (5), we get

$e^{b t} \frac{d C}{d t}+b C e^{b t} \geq m e^{b t}$

Integrating (6), we get

$$
C e^{b t} \geq e^{b t} \frac{m}{b}+b_{1}
$$

where $b_{1}$ is an integrating constant and initially when $t=0$ then $C(t) \geq C(0)$. Equation (7) becomes

$$
b_{1} \geq \frac{m}{b}-C
$$

Putting the value of $b_{1}$ in (7), we get

$$
\therefore C(t) \geq \frac{m}{b}
$$

Since $\boldsymbol{m}$ is the initial amount of carbon dioxide and that is positive and $b$ is always positive because the rate of absorbing carbon dioxide by phytoplankton is greater than the rate of emission of $\mathrm{CO}_{2}$ by zooplankton.

Therefore, $C(t) \geq 0$ at $t=0$ and $t \rightarrow \infty$.

Similarly, it can be proved that $P(t) \geq 0, Z(t) \geq 0$ and $D(t) \geq 0$ at $t=0$ and $t \rightarrow \infty$.

Therefore, $C(t) \geq 0, P(t) \geq 0, Z(t) \geq 0, D(t) \geq 0, \forall t \geq 0$.

Hence the lemma is verified.

\section{B. Equilibrium Points}

The equilibrium points of the model (1)-(4) can be obtained by setting $\frac{d \bar{C}}{d t}=0, \frac{d \bar{P}}{d t}=0, \frac{d \bar{Z}}{d t}=0, \frac{d \bar{D}}{d t}=0$. The system produces four dynamic equilibrium points $E_{i}(\bar{C}, \bar{P}, \bar{Z}, \bar{D})$, where $i=1,2,3,4$ and these are shown as

(i). $E_{1}(0,0,0,0)$, which is the trivial equilibrium point. 

(ii). $E_{2}(\bar{C}, 0,0, \bar{D})=E_{2}\left(\frac{m}{\psi_{3}}, 0,0, \frac{n}{\sigma_{4}}\right)$
(iii). $E_{3}(\bar{C}, \bar{P}, 0, \bar{D})=\left(\frac{c_{1}+m}{\left(\psi_{2} \bar{P}+\psi_{3}\right)}, \frac{c_{2}}{\varpi_{1}}, 0, \frac{n+\sigma_{1} \bar{P} \bar{C}-c_{3}}{\left(\sigma_{3} \bar{P}+\sigma_{4}\right)}\right)$

where $\varpi_{1}=\frac{\beta_{1} \bar{C}}{\alpha_{1}+D_{0}-\bar{D}}-\phi_{1}$, and $c_{1}, c_{2}, c_{3}$ are the constants.

(iv) $E_{4}(\bar{C}, \bar{P}, \bar{Z}, \bar{D})=\left(\frac{m-d_{1}}{\chi_{1}}, \frac{d_{2}}{\chi_{2}}, \frac{d_{3}}{\chi_{3}}, \frac{n+\sigma_{1} \bar{P} \bar{C}-d_{4}}{\left(\sigma_{2} \bar{Z}+\sigma_{3} \bar{P}+\sigma_{4}\right)}\right)$

where $\chi_{1}=\psi_{2} \bar{P}+\psi_{3}-\psi_{1} \bar{Z}, \chi_{2}=\frac{\beta_{1} \bar{C}}{\alpha_{1}+D_{0}-\bar{D}}-\phi_{1}-\phi_{2} \bar{Z}$,

$\chi_{3}=\frac{\beta_{2} \bar{P}}{\alpha_{2}+D_{0}-\bar{D}}-\gamma_{1}$, and $d_{1}, d_{2}, d_{3}, d_{4}$ are constants.

\section{Stability Analysis}

Now, the nonlinear differential equations (1)-(4) can be evaluated into the Jacobian matrix given as

$$
J=\left[\begin{array}{cccc}
a_{11} & -\psi_{2} C & \psi_{1} C & 0 \\
\frac{\beta_{1} P}{\alpha_{1}+D_{0}-D} & a_{22} & -\phi_{2} P & \frac{\beta_{1} C P}{\left(\alpha_{1}+D_{0}-D\right)^{2}} \\
0 & \frac{\beta_{2} Z}{\alpha_{2}+D_{0}-D} & a_{33} & \frac{\beta_{2} P Z}{\left(\alpha_{2}+D_{0}-D\right)^{2}} \\
\sigma_{1} P & \sigma_{1} C-\sigma_{3} D & -\sigma_{2} D & \left(-\sigma_{2} Z+\sigma_{4}\right)
\end{array}\right]
$$

where $i=1,2,3,4, \mathrm{a}_{11}=\psi_{1} Z-\psi_{2} P-\psi_{3}$,

$$
a_{22}=\frac{\beta_{1} C}{\alpha_{1}+D_{0}-D}-\phi_{1}-\phi_{2} Z, a_{33}=\frac{\beta_{2} P}{\alpha_{2}+D_{0}-D}-\gamma_{1}
$$

Theorem 1. The system (1)-(4) is stable at the equilibrium point $E_{1}$.

Proof. The Jacobian matrix (10) at the equilibrium point $E_{1}$ becomes

$$
J_{E_{1}(0,0,0,0)}=\left[\begin{array}{cccc}
-\psi_{3} & 0 & 0 & 0 \\
0 & -\varphi_{1} & 0 & 0 \\
0 & 0 & -\gamma_{1} & 0 \\
0 & 0 & 0 & -\sigma_{4}
\end{array}\right]
$$

After solving the characteristic equation $\left|J_{E_{1}}-I \lambda\right|=0$, we get

$\left(-\psi_{3}-\lambda_{1}\right)\left(-\varphi_{1}-\lambda_{2}\right)\left(-\gamma_{1}-\lambda_{3}\right)\left(-\sigma-\lambda_{4}\right)=0$

Therefore, the eigenvalues are

$\lambda_{1}=-\psi_{3}, \lambda_{2}=-\varphi_{1}, \lambda_{3}=-\gamma_{1}$ and $\lambda_{4}=-\sigma_{4}$

Here all the eigenvalues are negative. So $E_{1}$ is stable.

Theorem 2. The system (1)-(4) at the equilibrium point $E_{2}$ is a saddle point.

Proof. The Jacobian matrix (10) at the equilibrium point $E_{2}$ becomes

$$
J_{E_{2}}=\left[\begin{array}{cccc}
-\psi_{3} & -\frac{\psi_{2} m}{\psi_{3}} & \frac{\psi_{1} m}{\psi_{3}} & 0 \\
0 & \frac{\beta_{2} \frac{m}{\psi_{3}}}{\alpha_{1}+D_{0}-\frac{n}{\sigma_{4}}}-\phi_{1} & 0 & 0 \\
0 & 0 & -\gamma_{1} & 0 \\
0 & \sigma_{1} \frac{m}{\psi_{3}}-\sigma_{3} \frac{n}{\sigma_{4}} & \sigma_{2} \frac{n}{\sigma_{4}} & -\sigma_{4}
\end{array}\right]
$$

Similarly, the characteristic equation $\left|J_{E_{2}}-I \lambda\right|=0$ gives the eigenvalues

$$
\lambda_{1}=-\psi_{3}, \lambda_{2}=\frac{\beta_{2} m}{\psi_{3}\left(\alpha_{1}+D_{0}-\frac{n}{\sigma_{4}}\right)}-\varphi_{1}, \lambda_{3}=-\gamma_{1}, \lambda_{4}=-\sigma_{4} .
$$

Then the critical point $E_{2}$ is a saddle point and will be stable if $\lambda_{2}<0$.

Theorem 3. The system (1)-(4) is a saddle point or a stable point at the equilibrium point $E_{3}$.

Proof. The Jacobian matrix (10) becomes at $E_{3}$,

$$
J_{E_{3}}=\left[\begin{array}{cccc}
-\psi_{2} \bar{P}-\psi_{3} & -\psi_{2} \bar{C} & \psi_{1} \bar{C} & 0 \\
0 & a_{22} & a_{23} & b_{24} \\
0 & 0 & \frac{\beta_{2} \bar{P}}{\alpha_{2}+D_{0}-\bar{D}}-\gamma_{1} & 0 \\
0 & b_{42} & \frac{\sigma_{2} \bar{D}+\sigma_{1} \psi_{1} \bar{P} \bar{C}}{\psi_{2} \bar{D}+\psi_{3}} & -\sigma_{4}
\end{array}\right]
$$

where $a_{22}=\frac{\beta_{1} \bar{C}-\varphi_{1}\left(\alpha_{1}+D_{0}-\bar{D}\right)\left(\psi_{2} \bar{D}-\psi_{3}\right)-\beta_{1} \psi_{2} \bar{P} \bar{C}}{\left(\alpha_{1}+D_{0}-\bar{D}\right)\left(\psi_{2} \bar{D}-\psi_{3}\right)}$,

$a_{23}=\frac{-\varphi_{2} \bar{P}\left(\alpha_{1}+D_{0}-\bar{D}\right)\left(\psi_{2} \bar{D}-\psi_{3}\right)+\psi_{1} \beta_{1} \bar{C} \bar{P}}{\left(\alpha_{1}+D_{0}-\bar{D}\right)\left(\psi_{2} \bar{D}-\psi_{3}\right)}$,

$b_{24}=\frac{\beta_{1} \bar{C} \bar{P}}{\left(\alpha_{1}+D_{0}-\bar{D}\right)^{2}}, b_{42}=\frac{\sigma_{1} \psi_{3} \bar{C}-\sigma_{3} \psi_{2} \bar{D} \bar{P}-\sigma_{3} \psi_{3} \bar{D}}{\psi_{2} \bar{D}+\psi_{3}}$.

The characteristic equation $\left|J_{E_{3}}-I \lambda\right|=0$ gives the eigenvalues

$$
\lambda_{1}=-\psi_{2} \bar{P}-\psi_{3}, \quad \lambda_{2}=a_{22}, \quad \lambda_{3}=\frac{\beta_{2} \bar{P}}{\alpha_{2}+D_{0}-\bar{D}}-\gamma_{1},
$$

$\lambda_{4}=-\sigma_{4}$.

Here two cases arise as below:

Case-1: If $\lambda_{2}<0$ and $\lambda_{3}<0$, then the equilibrium point $E_{3}$ will be stable.

Case-2: If $\lambda_{2}>0$ and $\lambda_{3}>0$, then the equilibrium point $E_{3}$ will be an unstable saddle point. But under the parametric values used in this model, the equilibrium point will be stable.

Theorem 4. The system (1)-(4) at the equilibrium point $E_{4}$ 
is a saddle point.

Proof. The Jacobian matrix (10) at $E_{4}$ becomes

$$
J_{E_{4}}=\left[\begin{array}{cccc}
b_{11} & -\psi_{2} \bar{C} & \psi_{1} \bar{C} & 0 \\
0 & b_{22} & b_{23} & \frac{\beta_{1} \bar{C} \bar{P}}{\left(\alpha_{1}+D_{0}-\bar{D}\right)^{2}} \\
0 & 0 & -\gamma_{1} & \frac{\beta_{2} \bar{P} \bar{Z}}{\left(\alpha_{2}+D_{0}-\bar{D}\right)^{2}} \\
0 & 0 & 0 & -\left(\sigma_{1} \bar{Z}+\sigma_{4}\right)
\end{array}\right]
$$

where $\quad b_{11}=\psi_{1} \bar{Z}-\psi_{2} \bar{P}-\psi_{3}, \quad b_{22}=\frac{\beta_{1} \bar{C}+\psi_{2} \beta_{1} \bar{C} \bar{P}}{\mathrm{~b}_{11}\left(\alpha_{1}+D_{0}-\bar{D}\right)}$,

$b_{23}=-\sigma_{2} \bar{P}-\frac{\psi_{1} \beta_{1} \bar{C} \bar{P}}{\mathrm{~b}_{11}\left(\alpha_{1}+D_{0}-\bar{D}\right)}$

Further, the characteristic equation $\left|J_{E_{4}}-I \lambda\right|=0$ gives the eigenvalues

$$
\lambda_{1}=b_{11}, \lambda_{2}=b_{22}, \lambda_{3}=-\gamma_{1}, \lambda_{4}=-\left(\sigma_{1} \bar{Z}+\sigma_{4}\right) .
$$

Here two eigenvalues are negative and another two $\left(\lambda_{1} \&\right.$ $\lambda_{2}$ ) can be positive or negative. Therefore $E_{4}$ is a saddle point and may be stable if $\lambda_{1}, \lambda_{2}<0$.

\section{RESULTS AND DisCUSSION}

Numerical simulation is a familiar content to express the interaction of dynamic variables in graphical representation. Here to check the feasibility of our analysis concerning stability axioms, we use Maple coding. Some numerical computations have been driven by using these coding by choosing a set of parameter values given below:

Table 1. Parametric values of parameters used in the model.

$\begin{array}{clcl}\begin{array}{c}\text { Symbol } \\ m\end{array} & \text { Values } & \text { Symbol } & \text { Values } \\ \psi_{1} & 0.5 \mathrm{mgl}^{-1} \text { day }^{-1} & \beta_{2} & 0.33 \text { day }^{-1} \\ \psi_{2} & 0.51 \mathrm{lmg}^{-1} \mathrm{mg}^{-1} \mathrm{day}^{-1} & \alpha_{2} & 0.41 \mathrm{mgl}^{-1} \\ \psi_{3} & 0.1 \mathrm{day}^{-1} & \gamma_{1} & 0.01 \text { day }^{-1} \\ \beta_{1} & 0.35 \mathrm{day}^{-1} & n & 24 \mathrm{mgl}^{-1} \mathrm{day}^{-1} \\ \alpha_{1} & 0.51 \mathrm{mgl}^{-1} & \sigma_{1} & 0.652 \mathrm{mgl}^{-1} \mathrm{day}^{-1} \\ \varphi_{1} & 0.009 \mathrm{day}^{-1} & \sigma_{2} & 0.02 \mathrm{mgl}^{-1} \mathrm{day}^{-1} \\ \varphi_{2} & 0.41 \mathrm{mg}^{-1} \mathrm{day} y^{-1} & \sigma_{3} & 0.025 \mathrm{day}^{-1} \\ D_{0} & 30 \mathrm{mgl}^{-1} & \sigma_{4} & 3 \text { day }^{-1}\end{array}$

The conditions for the existence of interior equilibrium $E_{4}$ are satisfied under the parametric values and the numerical solutions in $E_{4}$ are given by

$$
\bar{C}=5.723, \quad \bar{P}=0.668, \quad \bar{Z}=0.198, \quad \bar{D}=8.173
$$

We see that Fig. 2 approach to $E_{4}$ asymptotically. For a sufficiently long period, the numerical simulation has been driven with some initial values of dynamical variables. At first phytoplankton population declined frequently due to the oxygen deficit and because of predation occurred by zooplankton. The result of the declination of the phytoplankton population and deficit oxygen is the decrement of the zooplankton population. On the other hand, the concentration of carbon dioxide has raised sharply in absence of phytoplankton and declined in the presence of phytoplankton. After a sufficiently long period, the system will acquaint a certain point and gain a stable condition at $E_{4}$.

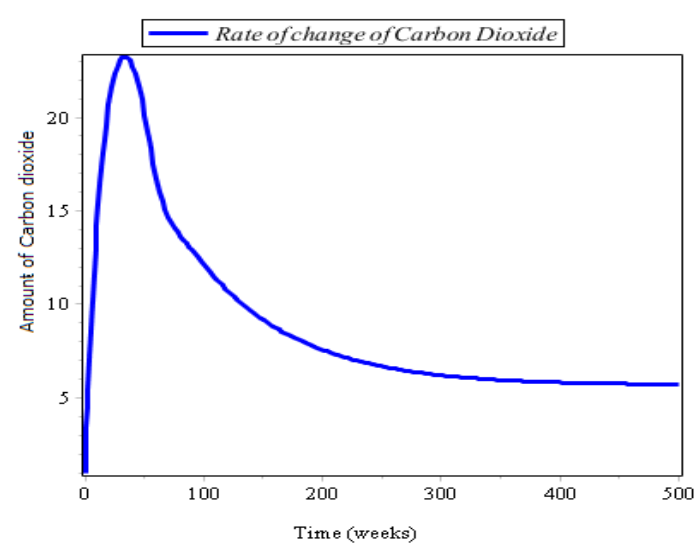

(a)

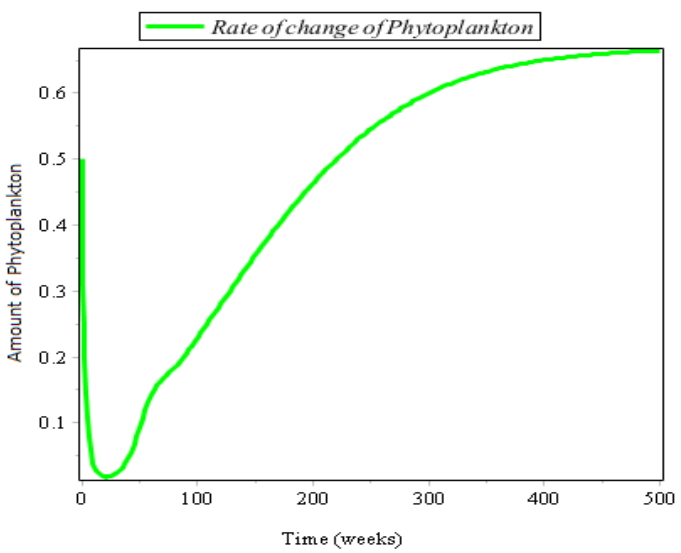

(b)

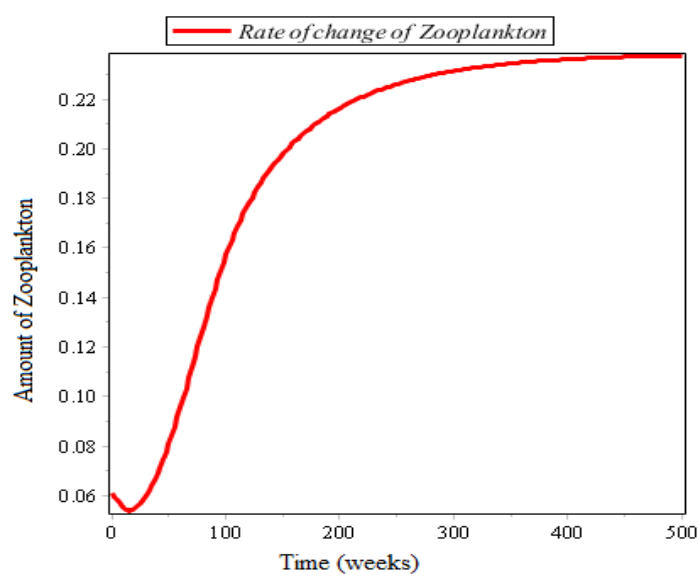

(c) 


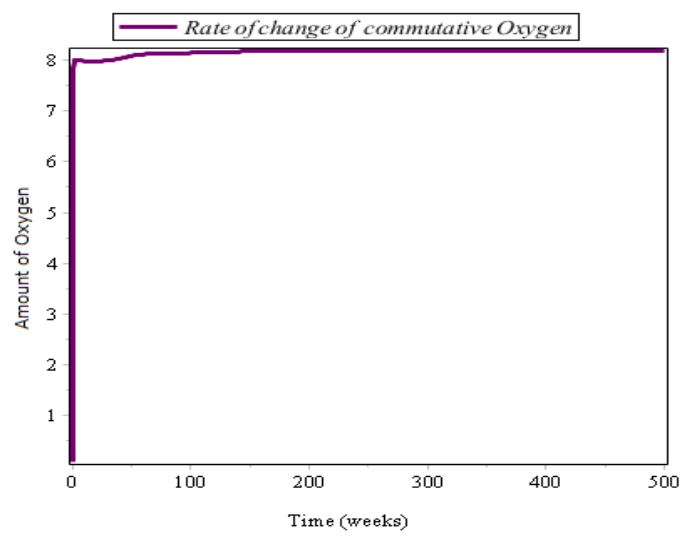

(d)

Fig.2 Stable interacting figures among the dynamical variables.

Carbon dioxide has a positive effect on phytoplankton. The growth rate of the planktonic ecosystem is changed due to the different values of carbon dioxide. When $m$ and $\psi_{1}$ are changed, the growth rate of phytoplankton, zooplankton, and oxygen are proportionally changed in the marine ecosystem. When the amount of carbon dioxide increases, the growth of phytoplankton increases. As a result, increasing phytoplankton promotes the growth of zooplankton and oxygen.

When $m$ promotes from $2.2 m g l^{-1} d_{a y}^{-1}$ to $2.8 m g l^{-1} d a y^{-1}$ and $\psi_{1}$ promotes from $0.100 \mathrm{lmg}^{-1} \mathrm{day}^{-1}$ to $0.120 \mathrm{lmg}^{-1} \mathrm{day}^{-1}$, the density of the saturated carbon dioxide increases in the ocean's water. But the density of the saturated carbon dioxide significantly increases when $m$ grows up to $3.2 \mathrm{mgl}^{-1} \mathrm{day}^{-1}$ and $\psi_{1}$ promotes to $0.150 \mathrm{lmg}^{-1} \mathrm{day}^{-1}$ which are shown in Fig. 3(a). When the density of saturated carbon dioxide increases in the ocean's water, the phytoplankton can easily perform the photosynthetic activity. As a result, the density of marine phytoplankton proportionally increases with the increase of the saturated carbon dioxide which is presented in Fig. 3(b). On the other hand, when the density of phytoplankton increases, it supplies food as well as oxygen for the zooplankton. As a result, the increasing density of phytoplankton proportionally increases the density of zooplankton and the density of dissolved oxygen which are shown respectively in Fig. 3 (c) and Fig. 3(d). Therefore, the simulations present that when the density of saturated carbon dioxide increases in the ocean's water, it enriches the density of plankton and indirectly contributes to producing more saturated oxygen.

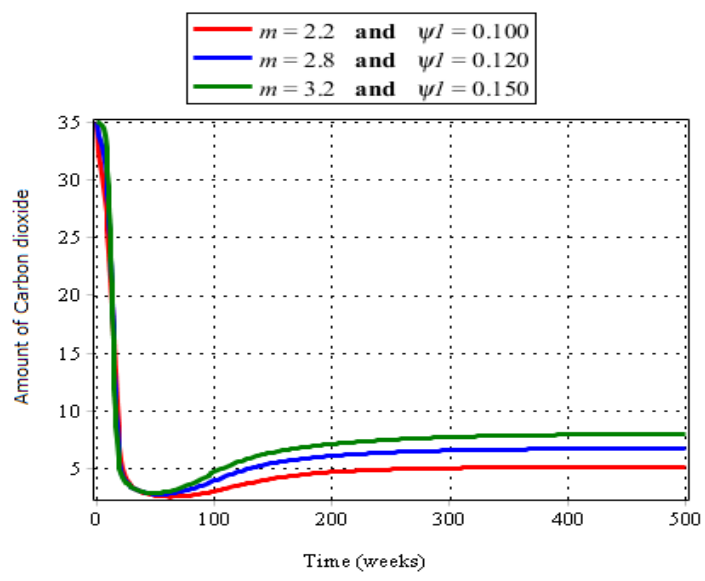

(a)

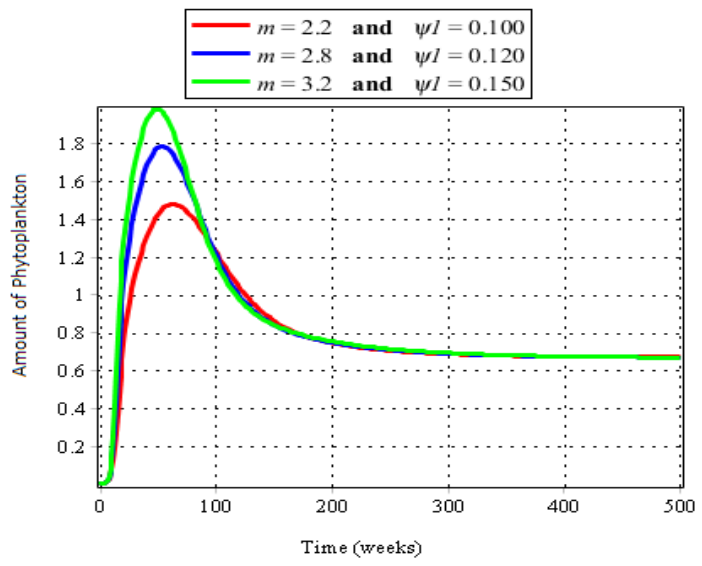

(b)

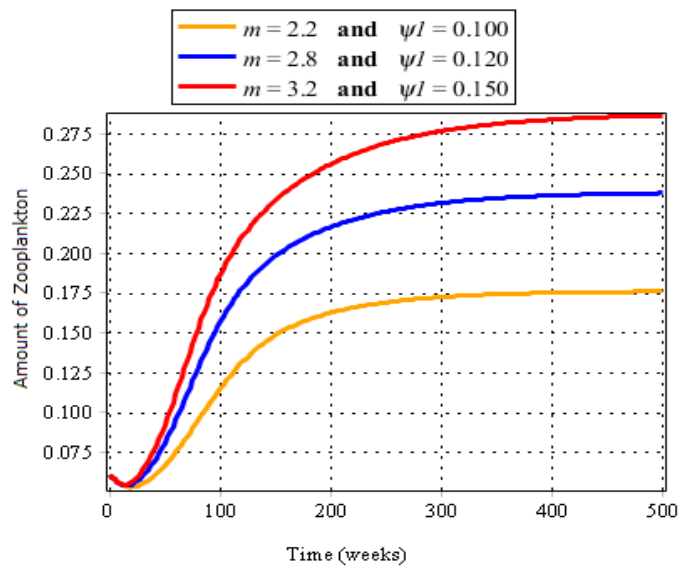

(c) 


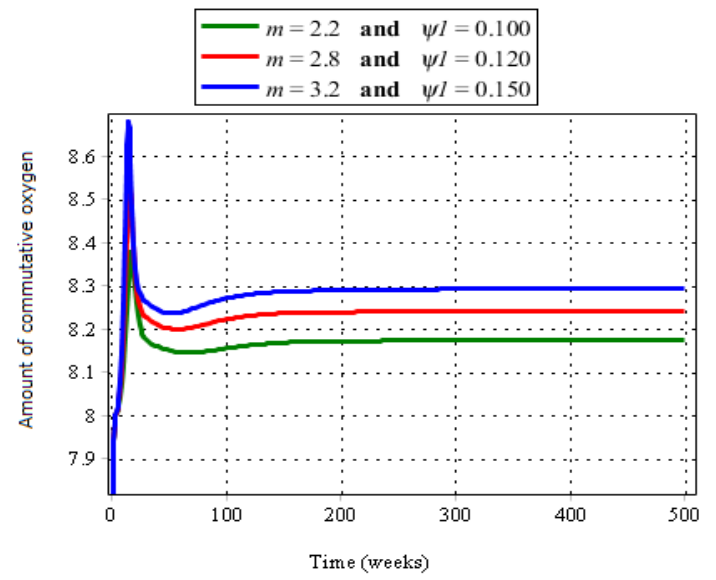

(d)

Fig.3 Growth rates of carbon dioxide, phytoplankton, zooplankton, and dissolved oxygen for the different values of $m$ and $\psi_{1}$.

\section{CONCLUSION}

This study is an environmental modeling work that describes the impacts of $\mathrm{CO}_{2}$ on marine plankton. A nonlinear mathematical model has been newly formulated. The study has been analyzed both analytically and numerically. The work aims to illustrate the potential impacts of carbon dioxide on the growth of marine plankton through mathematical modeling. We have performed the stability analysis at each obtained equilibrium point where one equilibrium point is stable and the other three are stable under some conditions. After investigating the simulations, we obtained that the density of phytoplankton proportionally increases with the increase in carbon dioxide. The study also showed that the production of oxygen and the absorption of carbon dioxide depend on the density of marine phytoplankton. The study represents the natural interactions among environmental factors. Therefore, it combines environmental dynamics with Applied Mathematics.

\section{References}

[1] Y. Sekerci and S. Petrovskii, "Mathematical Modeling of Plankton-Oxygen Dynamics under the Climate Change," Bulletin of Mathematical Biology, vol. 77(12), pp. 23252353, 2015.

[2] S. Mandal, M. S. Islam and M. H. A. Biswas, Modeling and Analytical Analysis of the Effect of Atmospheric Temperature to the Planktonic Ecosystem in Oceans, Lecture Notes in Networks and Systems. Springer, Singapore, vol. 137, pp. 131-140, 2021. https://doi.org/10.1007/978-981-15-6198-6 12

[3] Y. Destania, Jaharuddin and P. Sianturi, "Stability Analysis of Plankton Ecosystem Model Affected by Oxygen Deficit," Applied Mathematical Sciences, vol. 9(81), pp. 4043-4052, 2015.

[4] S. Khare, S. Kumar and C. Singh, "Modelling effect of the depleting dissolved oxygen on the existence of interacting planktonic population," S. Khare et al./Elixir Appl.Math, vol. 55, pp. 12739-12742, 2013.

[5] S. Mandal, M. S. Islam and M. H. A. Biswas, "Modeling the Potential Impact of Climate Change on Living beings near Coastal Areas," Modeling Earth Systems and Environment, 2020. https://doi.org/10.1007/s40808-020$\underline{00897-5}$

[6] E. M. Arnold and D.A. Voss, "Numerical behavior of zooplankton phytoplankton and phosphorus system," Ecol. Mode, vol. 13, pp. 183-193, 1981.

[7] A. M. Edwards and J. Brindley, "Zooplankton mortality and the dynamical behavior of plankton population models," Bulletin of Mathematical Biology, vol. 61, pp. 303- 339, 1999.

[8] S. Khare, O. P. Misra, C. Singh and J. Dhar, "Role of delay on planktonic Ecosystem in the presence of a toxic producing phytoplankton,” Int. J. of Diff. Equation, vol. 1, pp. 1-16, 2011.

[9] A. Chaturvedi and O.P. Misra, "Modelling Effects of eutrophication on the survival of fish population incorporating nutrient recycling," J. of Int. Acad. Of Phy. Sci., vol. 14, pp. 487-500, 2010.

[10] V. K. Naik and S. Manjapp, "Prediction of dissolved oxygen through mathematical modeling," Int. J. Environ. Res., vol. 4(1), pp. 153- 160, 2010.

[11] J. B. Shukla, A. K. Misra and P. Chandra, "Mathematical modelling and analysis of the depletion of dissolved oxygen in eutrophied water bodies affected by organic pollutants," Nonlinear Analysis: Real world Applications, vol. 9, pp. 1851- 1865, 2008.

[12] M. H. A. Biswas, M. R. Hossain and M. K. Mondal, "Mathematical Modeling Applied to Sustainable Management of Marine Resources," Procedia Engineering, vol. 194, pp. 337-344, 2017.

[13] M. H. A. Biswas, T. Rahman and N. Haque, "Modeling the Potential Impacts of Global Climate Change in Bangladesh: An Optimal Control Approach," J Fundam. Appl. Sci., vol. 8(1), pp. 1-19, 2016.

[14]K. Sardar, M. Hanif, M. Asaduzzaman and M. H. A. Biswas, "Mathematical Analysis of the Two Species Lotka-Volterra Predator-Prey Inter-specific Game Theoretic Competition Model," Advanced Modeling and Optimization, vol. 18(2), pp. 231-242, 2016.

\section{Authors Contribution}

Sajib Mandal carried out the model formulation, simulation, and overall arranging of the manuscript.

M. S. Islam has performed analytical analysis.

M. H. A Biswas has implemented the Algorithm and simulation. 